\title{
Validación de contenido de un catálogo de estímulos corporales visuales*
}

Validity Content of Stimuli Bodily Catalog

Recibido: febrero 10 de 2010 | Revisado: marzo 13 de 2011 | Aceptado: junio 13 de 2011

\author{
Rosa Margarita Zuvirie HernándeZ ** \\ Rosalía VÁzQUEZ ARÉvalo \\ Juan Manuel Mancilla DíaZ \\ Facultad de Estudios Superiores Iztacala, UNAM, México
}

JuAN José Cervantes NavarRete

Instituto Nacional de Psiquiatría Juan Ramón

de la Fuente Muñiz, México

SICI: 1657-9267(201303)12:1<185:VCCECV>2.0.TX;2-V

Para citar este artículo: Zuvirie, R. M., Vásquez, R., Mancilla, J. M. \& Cervantes, J. J. (2013). Validación de contenido de un catálogo de estímulos corporales visuales. Universitas Psychologica, 12(1), 185-193.

* Proyecto de Investigación en Nutrición, UNAMFES Iztacala.

** Avenida de los Barrios No.1. Los Reyes Iztacala. Tlalnepantla Estado de México. Teléfonos: 5623 11 13/15, Extensiones: 410 o 409. Fax: 53907604. E-mail: ixbalenka@yahoo.com.mx

\section{RESUMEN}

Parte de la investigación en Trastornos de la Conducta Alimentaria (TCA) se ha enfocado en la exhibición experimental del ideal de delgadez, donde se han empleado diferentes estímulos (Groesz et al., 2002), sin embargo, estos pocas veces son sometidos a procesos de validación. Por esto, el presente estudio tuvo como objetivo la validación de contenido de un catálogo de imágenes de mujeres delgadas, normopeso y obesas. Participaron ocho mujeres que fueron denominadas Jueces Expertos en TCA y 30 mujeres estudiantes de 19 a 25 años quienes fueron nombradas Jueces No Expertos. Se realizó una búsqueda en Internet de imágenes gratuitas de mujeres en tres categorías de peso: delgadez, normopeso y obesidad. De esta búsqueda resultaron 150 imágenes que se editaron. Posteriormente, fueron aleatorizadas en diapositivas para ser clasificadas por los jueces en delgadez, normopeso y obesidad. Se calculó el índice kappa para saber el acuerdo entre los Jueces Expertos, que reveló un nivel de coincidencia adecuado (kappa $=0.7-0.76$, $p<0.05)$. A continuación, se realizó un análisis de las coincidencias entre los Jueces Expertos y No Expertos, se obtuvieron 32 imágenes de delgadez, 38 de obesidad y 12 de normopeso con un porcentaje de acuerdo del $70 \mathrm{al}$ $100 \%$. La validación de contenido de este catálogo es una aproximación a la presentación experimental de estímulos que representan el ideal de delgadez. Palabras clave autores

Estímulos, validez de contenido, imágenes, delgadez, obesidad, normopeso.

Palabras clave descriptores

Psicología de la Salud, Alimentación, Validación

\section{A B S T R A C T}

Part of the research on eating disorders (ED) has focused on experimental presentation of thin ideal, with different stimuli (Groesz, et al., 2002); however, these rarely have been subjected to validation processes. Therefore, this study has aimed the content validity of an image catalog of thin, average weight and obese women. Participated 8 women who were called "Expert Judges" on ED and 30 female students aged 19 to 25 who were called "Non Expert Judges". We performed a search online of free images of women in three weight categories: thin, average weight and obesity. Of this search resulted 150 images which were edited. Subsequently images were randomized in slides to be categorized by judges in thin, average weight and obesity. We calculated the kappa index to find agreement among Expert Judges, this revealed adequate level of agreement (kappa $=0.7$ to $0.76, p<0.05)$. Then it was performed an analysis of agreement between Expert and Non Expert Judges, we obtained 32 Thinness, 38 Obesity and 12 Average weight images with an agreement percentage of 70 to $100 \%$. The content validity of this 
catalog is an approach of the experimental presentation of stimuli that represents the thin ideal.

Key words authors

Stimuli, content validity, images, thin, obesity, averageweight.

Key words plus

Health Psychology, Feeding, Validity.

\section{Introducción}

Desde hace mucho tiempo, el cuerpo femenino ha sido objeto de crítica y admiración, y se ha adaptado a los estándares impuestos por la moda, que magnifica u oculta las líneas y curvas femeninas, clasificándolas como adecuadas o inadecuadas de acuerdo a cada época. Así, a finales del siglo XIX, la moda tomó partido por cuerpos más delgados, y para principios del siglo XX, el tipo de vestimenta se hizo más ajustada para destacar al máximo las figuras esbeltas, tratando de suprimir todo aquello que no favorecía la silueta, quedando de esta forma instaurado un modelo de belleza que hoy conocemos como modelo ideal de delgadez o cultura proadelgazamiento (Toro, 1996).

La propagación de este modelo tiene su principal escaparate en ciertos medios masivos de comunicación (Groesz, Levine \& Murnen, 2002), donde el mensaje enviado es que la delgadez es deseable y admirable. Para internalizar este mensaje se requiere de un constante bombardeo que presumiblemente tiene un impacto sobre algunas mujeres (Dorian \& Garfinkel, 2001; Polivy \& Herman, 2004). Esto ha sido de gran interés en el campo de la investigación en Psicología, utilizando la exhibición experimental de estímulos prodelgadez para apreciar el efecto que estos tienen sobre la valoración corporal.

La literatura al respecto señala que quienes leen revistas de moda y prestan más atención a los comerciales relacionados con la apariencia muestran mayor preocupación por la delgadez, insatisfacción corporal, frustración con el peso, miedo a no pertenecer al estándar social y mayor riesgo de desarrollar sintomatología de un trastorno de la conducta alimentaria, que quienes leen otro tipo de publicaciones (Botta, 2003; Morry \& Staska, 2001; Turner, Hamilton, Jacobs, Angood \& Hovde, 1997). Al parecer, este tipo de publicidad tiene un efecto directo en los estados de ánimo, propiciando una inconformidad con su imagen corporal, particularmente entre mujeres jóvenes con altos niveles de internalización del modelo ideal de delgadez y tendencias a la comparación social, lo que se refiere a la predisposición que tienen algunas mujeres a comparar su figura corporal con las imágenes publicitarias de mujeres emaciadas (Birkeland et al., 2005; Brown \& Dittmar, 2005; Durkin \& Paxton, 2002; Halliwell, Dittmar \& Howe, 2005; Hargreaves \& Tiggemann, 2002; Tiggemann, Polivy \& Hargreaves, 2009; Yamamiya, Cash, Melnyk, Posovac \& Posovac, 2005). Es decir, la confrontación de la apariencia como un proceso subyacente, por el cual la publicidad puede incrementar la insatisfacción corporal (Dittmar \& Howard, 2004; Hargreaves $\&$ Tiggemann, 2004).

Sin embargo, aún no es claro este proceso; quizás la exhibición a estas imágenes idealizadas pueda tener solo un impacto temporal en algunas mujeres, pero, dada la difusión que tienen en la vida diaria, el efecto podría ser más duradero para otras (Hargreaves \& Tiggemann, 2004; Smeesters \& Mandel, 2006). Al parecer, hay una relación multidimensional entre la exposición a las imágenes ideales de los medios, las reacciones psicológicas a estos, las actitudes y conductas que reflejan el deseo por alcanzar estos ideales, lo que puede contribuir al deseo por perder peso (Cahill \& Mussap, 2007).

En términos metodológicos, los estímulos prodelgadez han sido expuestos a mujeres de diferentes edades, con o sin Trastornos de la Conducta Alimentaria (Cash, Cash \& Butters, 1983; Grogan, Williams \& Corner, 1996; Hamilton \& Waller, 1993; Heinberg \& Thompson, 1995). Los estímulos utilizados han sido desde 1 hasta 20, exhibiendo cuerpos desnudos digitalizados, en trajes de baño, con vestimenta de pasarelas, y presentados tanto en diapositivas, fotos, fragmentos de comerciales e incluso directamente de las revistas (Cattarin, Thompson, Thomas \& Williams, 2000; Crounch \& Degelman, 1998; Freske, 1998).

Los diseños experimentales que se han seguido incluyen mediciones pretest y/o postest y entre sujetos (Groesz et al., 2002). A pesar de la diversidad de los diseños, pocos protocolos se ocupan de someter a procedimientos de validación la serie de 
estímulos que se probarán. Uno de los métodos para validar una serie de estímulos es por medio de la validación de contenido, a través de la opinión de Jueces Expertos en el tema del constructo a validar (Pardo \& Ruiz, 2002). Por esto, la presente investigación tuvo como objetivo realizar la validación de contenido de un catálogo de imágenes de mujeres obesas, delgadas y normopeso, considerando la opinión de un grupo de mujeres tanto expertas como no expertas en el campo de investigación de los Trastornos de la Conducta Alimentaria, para lograr obtener aquellos estímulos que representen obesidad, delgadez y normopeso.

\section{Método}

\section{Participantes}

Participaron ocho mujeres especialistas: dos psiquiatras, dos psicólogas, dos nutricionistas y dos antropólogas físicas, que han trabajado en el campo de los Trastornos de la Conducta Alimentaria (TCA), quienes fueron llamadas Jueces Expertos, por una parte $y$, por otra, 30 mujeres estudiantes de nivel Licenciatura, con una media de 21.83 años $(D E=1.913$, rango $=19-26)$, residentes del Distrito Federal (20\%) y del Área Metropolitana (80\%), quienes fueron denominadas Jueces No Expertas en TCA.

\section{Procedimiento}

Para conformar el catálogo como primer paso se realizó la búsqueda de las imágenes gratuitas en la web (www.google.com), para elegirlas estas debían ajustarse a los siguientes criterios: ser modelos desconocidas, sin vestimenta extravagante, no aparecer en posturas sugerentes, estar en una postura de pie de frente y tenían que ser imágenes de libre acceso. La búsqueda de las imágenes se realizó separando las clasificaciones de peso corporal. Para obtener las imágenes de delgadez las palabras clave fueron: mujeres delgadas, modelos, pasarelas y colecciones de ropa. Para las imágenes de normopeso las palabras clave fueron: mujeres normopeso, peso ideal y peso adecuado. Finalmente, para las imágenes de obesidad las palabras clave fueron: ropa para gorditas, tallas extra, modelos talla extra, pasarelas talla extra y ropa en talla extra.

Como resultado de la búsqueda se obtuvieron 600 imágenes, de las cuales se seleccionaron 50 de cada categoría de peso corporal, es decir, aquellas que cubrían los criterios antes mencionados, quedando 50 imágenes de obesidad, 50 de normopeso y 50 de delgadez, sumando un total de 150 imágenes para el catálogo.

Posteriormente, estas imágenes se editaron dejando solo la silueta corporal en un fondo blanco para evitar distractores, luego todas fueron organizadas aleatoriamente en diapositivas, numeradas en la parte inferior derecha y exhibidas en conjunto durante 12.5 minutos, cada imagen fue expuesta durante 5 segundos. Una vez organizadas se sometieron a la opinión de los Jueces Expertos y No Expertos en TCA, quiénes las clasificaron como: delgadez, obesidad o normopeso.

Las siguientes instrucciones fueron dadas de manera individual a cada uno de los jueces:

En la siguiente presentación Power Point observarás una serie de fotografías de mujeres, por favor clasifícalas marcando con una "X" la opción de peso que mejor las identifique (delgadez, normopeso u obesidad).

\section{Resultados}

El análisis de resultados se inició con el grado de acuerdo entre el grupo de Jueces Expertos; para este grupo en particular se usó el índice kappa (véase Tabla 1), el cual reveló que al comparar a los Jueces Expertos dentro de sus diferentes profesiones tuvieron un nivel de coincidencia adecuado entre sí al categorizar las imágenes del catálogo $(\mathrm{kappa}=0.70-0.76, p<0.05$; porcentaje de acuerdo de 70-84 \%). Integrado a lo anterior, al comparar a los Jueces Expertos dentro de las mismas profesiones el análisis mostró un nivel de acuerdo medio (kappa $=0.42-0.70, p<0.05$; porcentaje de acuerdo $61-92 \%)$.

Para seleccionar las imágenes que conformarían el catálogo definitivo fue necesario calcular el 
TABLA 1

Índice kappa entre los Jueces Expertos comparados a través de su profesión

\begin{tabular}{|c|c|c|c|c|}
\hline \multicolumn{2}{|c|}{ Jueces } & \multirow{2}{*}{$\frac{\text { Índice kappa }}{0.42}$} & \multirow{2}{*}{$\begin{array}{c}\text { Significancia } \\
0.001\end{array}$} & \multirow{2}{*}{$\begin{array}{c}\text { Porcentaje de acuerdo } \\
72 *\end{array}$} \\
\hline \multirow{7}{*}{ Psicóloga 1} & Psicóloga 2 & & & \\
\hline & Antropóloga 1 & 0.55 & 0.001 & $70 *$ \\
\hline & Antropóloga 2 & 0.5 & 0.001 & 68 \\
\hline & Nutricionista 1 & 0.6 & 0.001 & $73 *$ \\
\hline & Nutricionista 2 & 0.3 & 0.001 & 51 \\
\hline & Psiquiatra 1 & 0.55 & 0.001 & $70 *$ \\
\hline & Psiquiatra 2 & 0.54 & 0.001 & 69 \\
\hline \multirow{6}{*}{ Psicóloga 2} & Antropóloga 1 & 0.55 & 0.001 & $70 *$ \\
\hline & Antropóloga 2 & 0.5 & 0.001 & 65 \\
\hline & Nutricionista 1 & 0.65 & 0.001 & $76^{*}$ \\
\hline & Nutricionista 2 & 0.55 & 0.001 & $71 *$ \\
\hline & Psiquiatra 1 & $0.7 * *$ & 0.001 & $80 *$ \\
\hline & Psiquiatra 2 & 0.65 & 0.001 & $76 *$ \\
\hline \multirow{5}{*}{ Antropóloga 1} & Antropóloga 2 & 0.6 & 0.001 & $72 *$ \\
\hline & Nutricionista 1 & $0.7 * *$ & 0.001 & $82 *$ \\
\hline & Nutricionista 2 & 0.31 & 0.001 & 48 \\
\hline & Psiquiatra 1 & 0.52 & 0.001 & 67 \\
\hline & Psiquiatra 2 & 0.6 & 0.001 & $71 *$ \\
\hline \multirow{4}{*}{ Antropóloga 2} & Nutricionista 1 & $0.73 * *$ & 0.001 & $82 *$ \\
\hline & Nutricionista 2 & 0.45 & 0.001 & 63 \\
\hline & Psiquiatra 1 & $0.7 * *$ & 0.001 & $78 *$ \\
\hline & Psiquiatra 2 & 0.64 & 0.001 & $76^{*}$ \\
\hline \multirow{3}{*}{ Nutricionista 1} & Nutricionista 2 & 0.45 & 0.001 & 61 \\
\hline & Psiquiatra 1 & $0.76 * *$ & 0.001 & $84^{*}$ \\
\hline & Psiquiatra 2 & 0.66 & 0.001 & $77 *$ \\
\hline \multirow{2}{*}{ Nutricionista 2} & Psiquiatra 1 & 0.46 & 0.001 & 68 \\
\hline & Psiquiatra 2 & 0.54 & 0.001 & $70 *$ \\
\hline Psiquiatra 1 & Psiquiatra 2 & 0.68 & 0.001 & $78 *$ \\
\hline
\end{tabular}

Nota. Los asteriscos $(* *)$ muestran los Índices kappa $>0.70$ y el asterisco $(*)$ muestra los porcentajes de acuerdo $>70 \%$.

Fuente: Pardo y Ruíz (2002).

porcentaje de acuerdo entre los Jueces Expertos y No Expertos al categorizar las imágenes dentro del catálogo, esto se logró mediante una regla de tres, que se ejemplifica a continuación:

$100 \%$ es $=30$ (coincidencias)

"X \%" es $=29$ (coincidencias)

Al realizar la operación el porcentaje que corresponde a 29 coincidencias es $96.6 \%$, esto en el caso particular de los Jueces No Expertos. Esta regla se aplicó desde 29 hasta 21 coincidencias.

$100 \%$ es $=8$ (coincidencias)

"X \%" es $=7$ (coincidencias)
Realizando la operación el porcentaje que corresponde a 7 coincidencias es $87.5 \%$, esto en el caso particular de los Jueces Expertos. Esta regla se aplicó desde 7 hasta 5 coincidencias.

Obtenidos los porcentajes de acuerdo entre los Jueces Expertos y No Expertos solo se seleccionaron aquellas imágenes que tuvieran coincidencias entre 70 y $100 \%$.

Al final de la categorización, el análisis de coincidencias reveló que los Jueces Expertos categorizaron un total de 108 imágenes con un porcentaje del 75 al $100 \%$ de coincidencia, de las cuales, hubo un 
TABLA 2

Categorización de las imágenes de acuerdo al criterio de los Jueces Expertos

\begin{tabular}{ccccc}
\hline \multirow{2}{*}{ Porcentaje de Acuerdo } & \multicolumn{4}{c}{ Categoría de las imágenes } \\
\cline { 2 - 5 } & Obesa & Delgada & Normopeso & Totales por porcentaje \\
\hline 100 & 36 & 8 & 2 & 46 \\
87.5 & 10 & 14 & 10 & 34 \\
75 & 2 & 16 & 10 & 28 \\
\hline Total por categoría & 48 & 38 & 22 & 108 \\
\hline
\end{tabular}

Nota: el porcentaje de acuerdo entre los jueces fue obtenido a partir de las coincidencias que marca el índice de kappa. Fuente: Pardo y Ruíz (2002).

TABLA 3

Categorización de las imágenes de acuerdo a la opinión de los Jueces No Expertos

\begin{tabular}{ccccc}
\hline \multirow{2}{*}{ Porcentaje de Acuerdo } & \multicolumn{4}{c}{ Categoría de las imágenes } \\
\cline { 2 - 5 } & Obesa & Delgada & Normopeso & Totales por porcentaje \\
\hline 100 & 7 & 1 & 1 & 9 \\
96.6 & 7 & 4 & 0 & 11 \\
93.3 & 6 & 7 & 1 & 14 \\
90 & 3 & 6 & 2 & 11 \\
86.6 & 1 & 6 & 1 & 8 \\
83.3 & 8 & 2 & 2 & 12 \\
80 & 1 & 4 & 1 & 6 \\
76.6 & 4 & 7 & 3 & 14 \\
73.3 & 2 & 3 & 2 & 7 \\
70 & 2 & 7 & 1 & 10 \\
\hline Total por categoría & 41 & 47 & 14 & 102 \\
\hline
\end{tabular}

Nota: el porcentaje de acuerdo entre los jueces fue obtenido a partir de las coincidencias que marca el índice de kappa. Fuente: Pardo y Ruíz (2002).

mayor número clasificadas como delgadas y obesas (véase Tabla 2).

El análisis de coincidencias reveló que los Jueces No Expertos categorizaron un total de 102 imágenes con un porcentaje del 70 al $100 \%$ de coincidencia, de las cuales hubo una mayor cantidad de imágenes clasificadas como delgadas y obesas (véase Tabla 3).

Posterior a la obtención de los porcentajes de acuerdo entre los Jueces, se procedió a identificar las imágenes para el catálogo final, las cuales fueron elegidas con base en la coincidencia entre ambos grupos de jueces, es decir, aquellas imágenes que fueron clasificadas de la misma forma tanto por los jueces expertos como por los no expertos.

El catálogo definitivo quedó conformado por 82 imágenes, de las cuales 38 están en la categoría de mujeres obesas; 32, en la clasificación de mujeres delgadas y, finalmente, 12 en la clasificación de mujeres normopeso (véase Apéndice 1).

\section{Discusión}

El modelo estético de delgadez tiene un impacto negativo sobre la percepción corporal femenina; su constante promoción a través de ciertos medios de comunicación, que envían el mensaje de que la delgadez es deseable y admirable (Groesz et al., 2002), ha desencadenado que las mujeres especialmente vulnerables, que presentan mayor insatisfacción corporal o una mayor interiorización del ideal de delgadez (Polivy \& Herman, 2004; Dorian \& Garfinkel, 2001), se vean afectadas por la exhibición de este modelo estético. 
Ahora bien, el campo de investigación en Psicología ha mostrado interés en examinar el efecto que tiene sobre la percepción corporal la exhibición experimental de estímulos prodelgadez, los cuales han sido de diferentes tipos y formas, sin embargo, pocos de ellos son seleccionados a partir de un procedimiento de validación de contenido adecuado. Por esto, la presente investigación brinda una aproximación a la validación de estímulos que pueden ser empleados para la exhibición experimental de figuras corporales delgadas, normopeso y obesas.

Una de las fortalezas del presente estudio radica en haber empleado un método sistemático para evaluar los estímulos en tres diferentes categorías de peso corporal. Por otra parte, se contó con la participación de jueces expertos y no expertos en TCA en la evaluación de las imágenes que conformaron el catálogo; se incluyeron estos grupos de jueces debido a que es importante contar con el criterio tanto de un grupo multidisciplinario de profesionales especialistas en TCA como la opinión de mujeres jóvenes de población general. De tal manera que se tuvo cierta fiabilidad sobre los estímulos que formaron el catálogo final y que posteriormente pueden ser empleados en los procedimientos experimentales.

Los hallazgos del presente estudio señalan que no hubo diferencias a través de las diferentes profesiones de los Jueces Expertos al clasificar las imágenes del catálogo; además, clasificaron más imágenes (46) con el $100 \%$ de acuerdo, comparado con los jueces no expertos (9 imágenes al $100 \%$ de acuerdo), no obstante la cantidad de imágenes clasificadas entre el 70 y $100 \%$ de acuerdo por ambos grupos de jueces, fue semejante (108 y 102 imágenes, respectivamente).

Al final de la evaluación de los jueces quedaron 82 imágenes para formar parte del catálogo final, el cual está compuesto en mayor medida por imágenes de mujeres delgadas (32) y obesas (38) y en menor número por imágenes de mujeres normopeso (12). Una forma de explicar por qué hay más imágenes de obesidad comparadas con las de delgadez y normopeso, puede atribuirse a que fue más sencillo clasificar las imágenes en categorías de peso corporal extremas. Además de que para los jueces fue más problemático evaluar una figura con un peso corporal adecuado o saludable, lo cual no resulta sencillo debido a que los jueces no están exentos de ser permeados por los valores culturales estéticos, por lo cual se tendría que considerar este sesgo como normativo de la cultura actual.

Ahora bien, la literatura señala que las mujeres atienden selectivamente más de forma automática a los cuerpos delgados que a los cuerpos obesos, lo que sugiere que hay un sesgo de atención (Glauert, Rhodes, Fink \& Grammer, 2010). Tanto hombres como mujeres califican los cuerpos delgados como más atractivos que los normales o con sobrepeso. Quizás esta idealización de los cuerpos delgados influye en la atención selectiva hacia estos (Glauert et al., 2010). Adicionalmente, existe evidencia que señala que la distorsión de la imagen corporal en las mujeres podría relacionarse con una relativa disfunción del hemisferio derecho cerebral que podría tener fuertes implicaciones en el desarrollo de la distorsión corporal y los TCA, al menos en las mujeres (Mohr, Porter \& Benton, 2007). Probablemente, estos sean elementos a tomar en cuenta cuando se hacen valoraciones de la percepción de figuras con diferente peso corporal, lo cual no es un proceso sencillo debido a los factores involucrados en esto.

El método sistemático para obtener la validación de contenido de los estímulos en este estudio puede ser aplicado no solo a la selección de imágenes que representen diferentes categorías de peso, sino que puede ser trasladado a la selección de otro tipo de estímulos que necesiten sustento metodológico para ser empleados en estudios experimentales.

Los jueces valoraron y eligieron los mejores estímulos para el catálogo de imágenes resultante de este estudio, de este último podemos decir que entre sus ventajas se encuentra que se puede usar una sola categoría para exhibición experimental, es decir, extraer solo las imágenes de delgadez o solo las de normopeso u obesidad, todo depende del objetivo que se pretenda cubrir. Los estímulos del catálogo se encuentran libres de distractores, ya que las modelos aparecen en un fondo blanco, lo cual las hace eficientes para valorar la reacción que provoca la silueta corporal de dichas imágenes. En conjunto o por separado el catálogo cubre 
el objetivo de mostrar experimentalmente siluetas corporales de diferente peso.

Finalmente, a pesar de las limitaciones del presente estudio, se cubrió el objetivo principal que consistía en dar validez de contenido a un catálogo de mujeres delgadas, normopeso y obesas, basado en la opinión y experiencia de un grupo de jueces expertos y no expertos en el área de los Trastornos de la Conducta Alimentaria.

\section{Referencias}

Birkeland, R., Thompson, J. K., Herbozo, S., Roehrig, M., Cafri, G. \& Van den Berg, P. (2005). Media exposure, mood, and body image dissatisfaction: An experimental test of person versus product priming. Body Image, 2(1), 53-61. doi:10.1016/j. bbr.2011.03.031

Botta, R. A. (2003). For your health? The relationship between magazine reading and adolescents' body image and eating disturbances. Sex Role, 48(9-10), 389-399. Recuperado el 15 de febrero de 2011, de http://www.ingentaconnect.com/content/klu/ sers/2003/00000048/F0020009/00464344

Brown, A. \& Dittmar, H. (2005). Think "thin" and feel bad: The role of appearance schema activation, attention level, and thin ideal internalization for young women's responses to ultra-thin media ideals. Journal of Social and Clinical Psychology, 24(8), 1088-1113. Recuperado el 27 de noviembre de 2010, de http://www.nedic.ca/pledge/articles/ Think\%20thin\%20and\%20feel\%20bad.pdf

Cahill, S. \& Mussap, A. J. L. (2007). Emotional reactions following exposure to idealized bodies predict unhealthy body change attitudes and behaviors in women and men. Journal of Psychosomatic Research, 62(6), 631-639. doi:10.1016/j.jpsychores.2006.11.001

Cash, T. F., Cash, D. W. \& Butters, J. W. (1983). Mirror, mirror, on the wall? Contrast effects and selfevaluations of physical attractiveness. Personality and Social Psychology Bulletin, 9(3), 351-358. doi: 10.1177/0146167283093004

Cattarin, J. A., Thompson, J. K., Thomas, C. \& Williams, R. (2000). Body image, mood, and televised images of attractiveness: The role of social com- parison. Journal of Social and Clinical Psychology, 19(2), 220-239. Recuperado el 2 de septiembre de 2009, de http://cat.inist.fr/?aModele=afficheN\& cpsidt $=1437510$

Crounch, A. \& Degelman, D. (1998). Influence of female body images in printed advertising on selfratings of physical attractiveness by adolescent girls. Perceptual and Motor Skills, 87(2), 585-586. Recuperado el 4 de abril de 2010, de http://www. ncbi.nlm.nih.gov/pubmed/9842606

Dittmar, H. \& Howard, S. (2004). Thin ideal internalization and social comparison tendency as moderator of media models' impact on women's body-focused anxiety. Journal of Social and Clinical Psychology, 23(6), 768-791. doi: 10.1521/jscp.23.6.768.54799

Dorian, L. \& Garfinkel, P. E. (2001). Culture and body image in Western society. Eating and Weight Disorders, 7(1), 1-19. Recuperado el 26 de marzo de 2008, de http://www.ncbi.nlm.nih.gov/pubmed/11930981

Durkin, S. J. \& Paxton, S. J. (2002). Predictors of vulnerability to reduced body image satisfaction and psychological wellbeing in response to exposure to idealized female media images in adolescent girls. Journal of Psychosomatic Research, 53(5), 995-1005. Recuperado el 7 de julio de 2009, de http://www.sciencedirect.com/science/article/pii/ S0022399902004890

Freske, P. (1998). Possible moderators of the impact of media exposure on affect and body satisfaction in college females. Tesis de Maestría inédita, Washington State University, Vancouver, W.A. Recuperado el 23 de marzo de 2010, de http:// books.google.com.mx/books/about/Possible_ moderators_of_the_impact_of_med.html?id=7WXNwAACAAJ\&redir_esc $=y$

Glauert, R., Rhodes, G., Fink, B. \& Grammer, K. (2010). Body dissatisfaction and attentional bias to thin bodies. International Journal of Eating Disorders, 43(1), 42-49. doi: 10.1002/eat.20663

Groesz, L. M., Levine, M. P. \& Murnen, S. K. (2002). The effect of experimental presentation of thin media images on body satisfaction: A meta-analytic review. International Journal of Eating Disorders, 31(1), 1-16. Recuperado el 3 de enero de 2011, de http://www.ncbi.nlm.nih.gov/pubmed/11835293 
Grogan, S., Williams, Z. \& Conner, M. (1996). The effects of viewing same-gender photographic models on body esteem. Psychology of Women Quarterly, 20(4), 569-575. doi: 10.1111/j.1471-6402.1996. tb00322.x

Halliwell, E., Dittmar, H. \& Howe, J. (2005). The impact of advertisements featuring ultra-thin or averagesize models on women with a history of eating disorders. Journal of Community 8 Applied Social Psychology, 15(5), 406-413. doi: 10.1002/casp.831

Hamilton, K. \& Waller, G. (1993). Media influences on body size estimation in anorexia and bulimia: An experimental study. British Journal of Psychiatry, 162, 837-840. doi: 10.1192/bjp.162.6.837

Hargreaves, D. \& Tiggemann, M. (2002). The effect of television commercials on mood and body dissatisfaction: The role of appearance schema activation. Journal of Social and Clinical Psychology, 21(3), 287308. doi: 10.1521/jscp.21.3.287.22532

Hargreaves, D. A. \& Tiggemann, M. (2004). Idealized media images and adolescent body image: "Comparing" boys and girls. Body Image, 1(4), 351- 361. doi: 10.1016/j.bodyim.2004.10.002

Heinberg, L. B. \& Thompson, J. K. (1995). Body image and televised images of thinness and attractiveness: A controlled laboratory investigation. Journal of Social and Clinical Psychology, 14(4), 325-338. doi: 10.1521/jscp.1995.14.4.325

Mohr, C., Porter, G. \& Benton, C. P. (2007). Psychophysics reveals a right hemispheric contribution to body image distortions in women but not men. Neuropsychologia, 45(13), 2942-2950. doi:10.1016/j. neuropsychologia.2007.06.001

Morry, M. M. \& Staska, S. L. (2001). Magazine exposure: Internalization, self-objectification, eating attitudes, and body satisfaction in male and female. Canadian Journal of Behavioral Science, 33(4), 269-279. Recuperado el 17 de agosto de 2008, de http://psycnet.apa.org/index.cfm?fa=buy. optionToBuy\&id=2002-17969-006

Pardo, M. A. \& Ruiz, D. M. A. (2002). SPSS 11. Guía para el análisis de datos. Madrid: McGraw-Hill.

Polivy, J. \& Herman, C. P. (2004). Socio-cultural idealization of thin female body shapes: An introduction to the special issue on body image and eating disorders. Journal of Social Eु Clinical Psychology, 23(1), 1-6. doi:10.1037/0033-2909.134.3.460

Smeesters, D. \& Mandel, N. (2006). Positive and negative media image effects on the self. Journal of Consumer Research, 32(4), 576-582. doi: 10.1086/500489

Tiggemann, M., Polivy, J. \& Hargreaves, D. (2009). The processing of thin ideals in fashion magazines: $A$ source of social comparison or fantasy? Journal of Social and Clinical Psychology, 28(1), 73-93. doi: 10.1521/jscp.2009.28.1.73

Toro, J. (1996). El cuerpo como delito: anorexia, bulimia, cultura y sociedad. Barcelona: Ariel.

Turner, S. L., Hamilton, H., Jacobs, M., Angood, L. M. $\&$ Dwyer, D. H. (1997). The influence of fashion magazines on the body image satisfaction of college women: An exploratory analysis. Adolescence, 32(127), 603-614. Recuperado el 15 de octubre de 2009, de http://www.ncbi.nlm.nih.gov/pubmed/9360734

Yamamiya, Y., Cash, T. F., Melnyk, S. E., Posovac, H. D. \& Posovac, S. S. (2005). Women's exposure to thin and beautiful media images: Body image effects of media ideal internalization and impact reduction interventions. Body Image, 2(1), 74-80. Recuperado el 28 de junio de 2010, de http://www. ncbi.nlm.nih.gov/pubmed/18089176 
Apéndice 1

Muestras de imágenes que conforman el catálogo

Delgadez

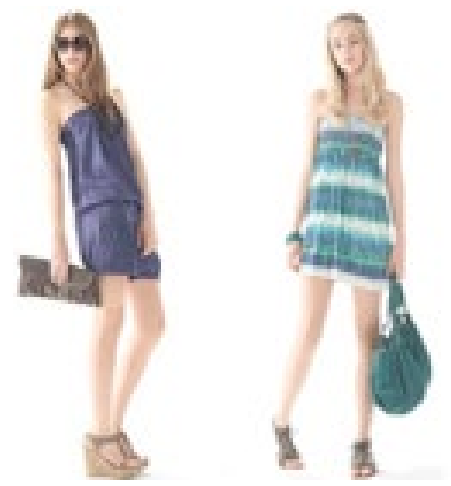

Normopeso

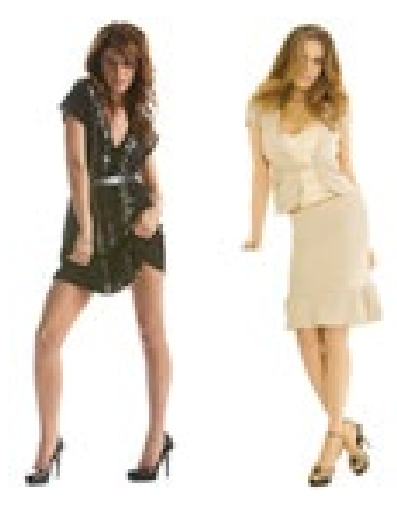

Obesidad

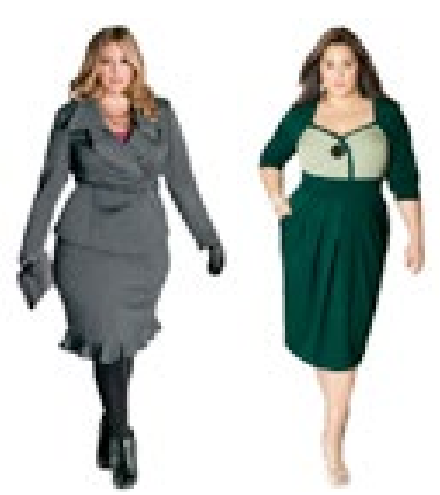


